\title{
Changes in pharyngeal airway space and soft tissue after maxillary advancement and bimaxillary surgery
}

\author{
Erika Franco Freire ${ }^{1}$, Francisco Wagner Vasconcelos Freire Filho ${ }^{2}$, Heloisa Cristina Valdrighi ${ }^{2}$, \\ Viviane Veroni Degan², Silvia Amélia Scudeler Vedovello²
}

${ }^{1}$ Centro Universitário Hermínio Ometto - UNIARARAS, School of Dentistry, Area of Orthodontics, Araras, SP, Brazil 2Universidade de Fortaleza - UNIFOR, School of Dentistry, Department of Oral and Maxillofacial Surgery, Fortaleza, Ceará, CE, Brazil

\begin{abstract}
Aim: To evaluate changes in pharyngeal airway space (nasopharynx and oropharynx), soft palate and lingual vallecula after maxillary advancement surgery and maxillary advancement and mandibular setback surgery (bimaxillary surgery). Methods: Twenty Class III adult patients were included in the study. Ten patients were treated with maxillary advancement and ten with bimaxillary surgery (maxillary advancement and mandibular setback). Cephalometric landmark measurements were recorded at 3 different time intervals: pre-surgical, post-surgical and six months after surgery. Data collected were subjected to one-way ANOVA $(p<0.05)$. Results: Nasopharyngeal airway space increased after maxillary advancement and decreased after bimaxillary surgery. There was increase in oropharyngeal dimensions in the region around the uvula and loss of space for lingual vallecula, while in Group 2 there was diminished space for both uvula and vallecula. The uvula and vallecula were moved forward in Group 1, whereas these structures were moved in the posterior direction in Group 2. Conclusions: After maxillary advancement surgery there was an increased in space in the nasopharyngeal region. The oropharyngeal region related to the uvula presented an increase in space, whereas there was a reduction in relation to the lingual vallecula. The uvula and lingual vallecula were moved forward. Bimaxillary surgery promoted a reduction in the nasopharyngeal and oropharyngeal regions as regards both the uvula and lingual vallecula. The uvula and lingual vallecula were moved in the posterior direction.
\end{abstract}

Keywords: orthognathic surgery; pharynx; palate, soft.

\section{Introduction}

Received for publication: March 04, 2014 Accepted: May 29, 2014

Correspondence to: Sílvia Amélia Scudeler Vedovello UNIARARAS - Av. Dr. Maximiliano Baruto 500 Jardim Universitário CEP: 13607-339 Araras, SP, Brasil Phone: +55 1935431423 E-mail: silviavedovello@gmail.com
Dentofacial deformities treated by orthognathic surgery may influence functional soft tissue components including the tongue and pharyngeal airway ${ }^{1-6}$.

Soft palate, tongue and hyoid bone are directly or indirectly attached to each other, so that maxillary and mandibular movement affects these tissues by changing pharyngeal airway ${ }^{2-3}$.

Mandibular and maxillary advancement may offer larger space in the pharyngeal airways ${ }^{7-9}$, whereas mandibular setback surgery may result in reduction of the respiratory area in this region ${ }^{3,7}$.

A combination of mandibular setback and maxillary advancement are the procedures generally used for correction of severe Class III discrepancies ${ }^{10}$. However, studies have warned about the risk of orthognathic surgery effects for the correction 
of mandibular prognathism leading to reduction in pharyngeal airway space ${ }^{3,11}$.

Reduction in airway space may contribute to the development of obstructive sleep apnea ${ }^{1,3,8,12}$.

The aim of this study was to evaluate changes in pharyngeal airway space (nasopharynx and oropharynx), uvula and lingual vallecula after maxillary advancement surgery, and maxillary advancement and mandibular setback surgery. The main hypothesis is that the pharyngeal space increases with advancement of the maxilla, and diminishes with the combined maxillary advancement and mandibular setback surgery.

\section{Material and methods}

This study was approved by the institutional Research Ethics (Process \#999/2011) and the research subjects signed an informed consent form prior to their enrollment.

A retrospective study was conducted. The sample comprised 20 individuals of both genders $(11 \mathrm{men}$ and 9 women), age-range between 25 and 30 years (mean age 27 years and 5 months). Inclusion criteria were as follows: adult patients with skeletal and dental Class III deformity, mesiofacial skeletal pattern defined by the Frankfortmandibular plane (FMA) angle, between $21^{\circ}$ and $29^{\circ}$, according to Tweed; permanent dentition and posterior teeth; who had been submitted to orthognathic surgery for the correction of maxillary retrusion or combined surgery (maxillary advancement and mandibular setback) for the correction of skeletal Class III malocclusion. All patients used internal rigid fixation as a form of osteosynthesis.

The following exclusion criteria were applied: previous orthognathic surgery, additional surgical procedures, such as rhinoplasty, infraorbital grafts, craniofacial anomalies, posttraumatic deformity, obstructive sleep apnea and systemic diseases that could affect the stomatognathic system.

Forty lateral teleradiographs of the head were obtained from the archives comprising files belonging to the team of surgeons who performed the same surgical protocol. For each individual in the sample were analyzed the teleradiographs taken at two time intervals: immediate pre-surgical ( $\mathrm{t} 1)$ and post-surgical after six months ( $\mathrm{t} 2)$. In the initial and final cephalograms ( $\mathrm{t} 1$ and $\mathrm{t} 2)$ lines and cephalometric points were marked (Fig. 1 and Chart 1).

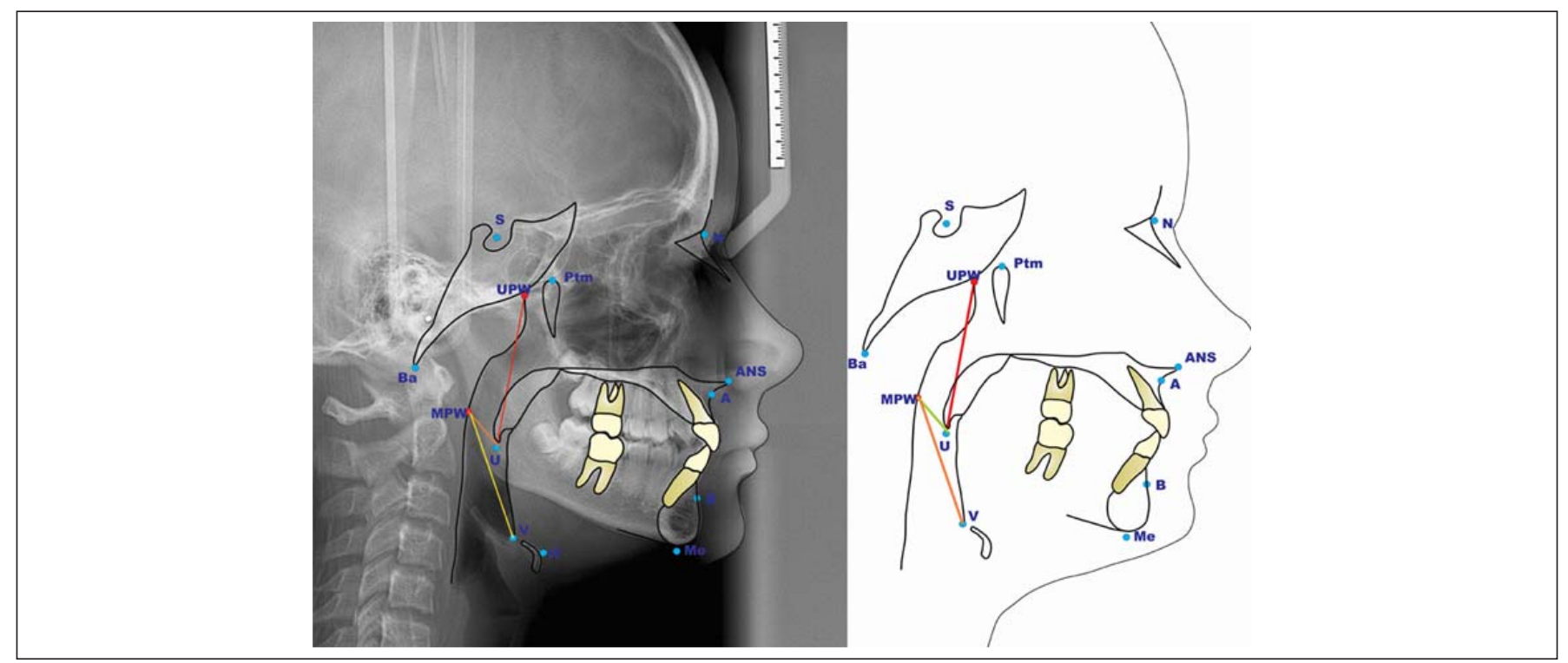

Fig. 1.Cephalometric variables used in the study

Chart 1. Cephalometric points and lines used in the study

\begin{tabular}{|ll|}
\hline Cephalometric & Description \\
Points and lines & Nasion \\
S & Sella \\
Ba & Basion \\
Ptm & Pterigomaxillary Fissure \\
ANS & Anterior Nasal Spine \\
A & Most posterior point of the maxillary alveolar bone concavity \\
B & Most posterior point of the mandibular alveolar bone concavity \\
Me & Mentum \\
U & Uvula: Uvula, the tip of the uvula \\
V & Vallecula: the intersection of the epiglottis and the base of the tongue \\
UPW & Linear measurement of the superior pharyngeal wall: Intersection between line Ptm-Ba and the posterior pharyngeal wall \\
MPW & Linear measurement of the mid-pharyngeal wall: Intersection between perpendicular line of Ptm-Ba at U and the posterior pharyngeal wall \\
\hline
\end{tabular}


The patients were divided into two groups. Group 1 $(\mathrm{n}=10)$, composed by individuals subjected to orthodonticsurgical treatment for maxillary advancement (G1) and Group $2(n=10)$, composed by individuals subjected to orthodonticsurgical treatment for maxillary advancement and mandibular setback (G2). The movements were solely advances and setbacks and therefore they did not cause clockwise or anticlockwise rotations.

The 40 radiographs were randomly disposed, and marking and measurements of the points were performed twice by the same investigator. The results were compared to obtain correlation coefficients $\left(\mathrm{r}^{2}\right)$.

To evaluate the results, the pharyngeal airway space was divided into 2 vertical levels (nasopharynx and oropharynx). To delimit these structures the cephalometric variables from Chart 2 were used.

Descriptive statistical analysis of the measurements of Groups G1 and G2 was performed, and for comparisons between means of the groups, one-way ANOVA was used, with a $5 \%$ significance level.

Chart 2. Division of the pharyngeal airway into nasopharynx and oropharynx.

\begin{tabular}{|ll|}
\hline Region & Measurements \\
Nasopharynx & \\
Nasopharyngeal airway & UPW-U \\
Oropharynx & \\
Oropharyngeal airway & MPW-U \\
& MPW-V \\
\hline
\end{tabular}

\section{Results}

For the nasopharyngeal region in Group 1, a gain of $+2.72 \mathrm{~mm}$ in anteroposterior space was observed (MPW-U). In Group 2, there was a loss of $-1.27 \mathrm{~mm}$ in anteroposterior space in the nasopharyngeal region (MPW-U) (Table 1). Statistically significant difference between the groups was found with reference to alterations in airway space in the nasopharynx area, (Table 1)
In the oropharyngeal region in Group 1 occurred a +1.25 $\mathrm{mm}$ gain in anteroposterior space (MPW-U) and $-2.84 \mathrm{~mm}$ loss of space in the MPW-V line. In Group 2 a reduction in anteroposterior space occurred in the oropharyngeal region (-1.42 mm in MPW-U and $-1.32 \mathrm{~mm}$ in MPW-V). Statistically significant difference was obtained with reference to alterations in airway space in the oropharynx area between the groups in uvula region. Statistically significant difference between the groups was not found with reference to alterations in airway space in the oropharynx area. For both groups occurred a reduction in airway space in the oropharynx area (Table 1).

Uvula and lingual vallecula moved forwards $(+0.57$ $\mathrm{mm}$ and $+1.57 \mathrm{~mm}$ respectively) in Group 1 while in Group 2 the structures were moved in a posterior direction $(-1.57$ $\mathrm{mm}$ and $-3.12 \mathrm{~mm}$ ). Statistically significant difference between the groups was not obtained with reference to the movement of uvula, while it was observed to the movement of lingual vallecula (Table 2).

\section{Discussion}

Orthognathic surgery has regularly been used to correct dentofacial deformities. Surgical procedures that modify the relationship of the bony base may bring about changes both in facial esthetics and in the component structures of the craniofacial complex and its functions. Changes in pharyngeal airway space may occur as a result of these procedures $^{2-4,13}$.

Studies that evaluated posterior airway space after orthodontic and orthopedic treatment with rapid maxillary expansion and with anterior traction of the maxilla referred to an increase in pharyngeal airway space, both in the nasopharynx and oropharynx ${ }^{14-16}$

In this study, the patients subjected to maxillary advancement surgery, had the nasopharynx increased. In the oropharyngeal region there was an increase in relation to the uvula and a reduction in relation to the lingual vallecula ${ }^{3,5,13}$. The interesting point is that as far as orthopedic maxillary

Table 1. Mean value $(\mathrm{mm})$ of changes in the nasopharyngeal and oropharyngeal space.

\begin{tabular}{|c|c|c|c|c|c|}
\hline \multirow[b]{2}{*}{ Group } & \multicolumn{4}{|c|}{ Mean of alterations in Nasopharynx and Oropharynx } & \multirow[b]{2}{*}{$p$} \\
\hline & Region & Mean & SD & Minimum - Maximum & \\
\hline Group 1 & UPWU & 2.72 & 3.34 & $-3.09-7.59$ & 0.026 \\
\hline Group 2 & Nasopharynx & -1.27 & 3.96 & $-6.02-4.93$ & \\
\hline Group 1 & MPWU & 1.25 & 2.97 & $-3.40-6.31$ & 0.049 \\
\hline Group 2 & Oropharynx & -1.42 & 2.68 & $-5.00-2.38$ & \\
\hline Group 1 & MPWV & -2.84 & 2.32 & $-6.03-1.25$ & 0.553 \\
\hline Group 2 & Oropharynx & -1.32 & 7.60 & $-14.44-14.21$ & \\
\hline
\end{tabular}

Table 2. Mean amount of movement of the uvula and lingual vallecula structures in millimeters.

\begin{tabular}{lllcl|llll}
\hline Group & \multicolumn{3}{c|}{ Movement of the uvula } & \multicolumn{4}{c}{ Movement of the lingual vallecula } \\
& Mean & SD & Minimum - Maximum & $\mathbf{p}$ & Mean & SD & Minimum - Maximum & p \\
Group 1 & 0.57 & 1.86 & $-2.53-3.20$ & 0.077 & 1.57 & 3.04 & $-2.50-8.00$ & 0.006 \\
Group 2 & -1.59 & 3.12 & $-5.83-2.89$ & & -3.12 & 3.72 & $-8.24-4.37$ \\
\hline
\end{tabular}


advancement is concerned, the nasopharynx gains space but the oropharynx tends to undergo positive changes gain or remains unaltered, whereas in the present study, the result was negative. One could suppose that this factor was caused due to the nature of the procedure, since orthopedics results in gradual alterations, whereas surgery has an immediate result, and may stretch the structures surrounding the operated region. In addition, the orthopedic patient is in the active growth stage and the surgical patient is already full grown. Another hypothesis would be the absence of the maxillary disjunction procedure in this study.

It was observed in this study that bimaxillary surgery promoted loss of pharyngeal airway space of the nasopharynx and oropharynx, a fact also reported by other authors ${ }^{3,7,10}$, but with higher values. Another study pointed out that the pharyngeal airway space and soft palate were moved to a significant extent in the mandibular setback ${ }^{17}$. The former was changed in the retropalatine direction and in the retrolingual direction; the latter underwent an increase in length.

Studies have shown that in bimaxillary surgeries ${ }^{7,13}$ the mandible underwent a setback and the maxilla an increase. A decrease occurred in the soft palate area and at the base of the tongue. This result demonstrated that, even advancing the maxilla, when combined with mandibular setback, there is a decrease in pharyngeal airway space. The backward movement of the soft palate and the tongue may invade the pharyngeal airway space and is a predisposing factor for obstructive sleep apnea ${ }^{8}$. This study demonstrated that with mandibular setback surgery, even associated with maxillary advancement, the lingual vallecula and uvula are moved in a backward direction. Thus, the oropharynx and nasopharynx airway spaces are diminished. Therefore, the professional should be aware of the risk of development of obstructive sleep apnea syndrome after bimaxillary surgeries in Class III patients.

In individuals with obstructive sleep apnea, the length of the soft palate may be increased, compared with the individuals that do not present this disturbance and they present reduction in the dimensions of the nasopharynx and oropharynx ${ }^{18}$. Therefore, one should be cautious about surgical movements, even in individuals who do not present signs and symptoms of apnea, in order to avoid elongation by displacement of this structure, and the reduction in oropharyngeal and nasopharyngeal space, thereby hindering the passage of air.

The presence of other predisposing factors for the obstructive sleep apnea syndrome must also be observed in candidates for mandibular setback surgery, such as overweight, short neck, tongue volume, wide uvula and excessive amount of soft tissue around the nasopharyngeal region, which may favor the development of the syndrome ${ }^{19-20}$. In addition, other factors such as the facial type and unsatisfactory neuromuscular adaptation should also be considered ${ }^{15}$.

Posterior movement of bony bases may diminish the posterior area of the tongue and retropalatine area, even when the maxilla has been advanced, causing reduction in the nasopharyngeal space ${ }^{21-22}$. Therefore, mandibular setback may potentially diminish this region and generate respiratory disorders, particularly if the individual does not have sufficient neuromuscular adaptation to compensate reduction in the size of space. Combined maxillary and mandibular surgeries may be used for the correction of accentuated anteroposterior discrepancies to minimize the side effects on the pharyngeal airway space ${ }^{22}$.

Based on the observations in this study, maxillary advancement surgery in skeletal Class III patients must be emphasized because little space is lost in the oropharynx and there is gain in the nasopharynx. Caution is strongly recommended for performing bimaxillary surgery in individuals with respiratory diseases, since there is a considerable loss of pharyngeal airway space. It should also be observed that even when advancing the maxilla, mandibular setback seems to influence the amount of functional airway space.

It was concluded that after maxillary advancement surgery there was an increase in the space of the nasopharyngeal region. The oropharyngeal region related to the uvula presented an increased space, whereas there was a decrease in relation to the lingual vallecula. The uvula and lingual vallecula were moved forward. Bimaxillary surgery promoted a reduction in the nasopharyngeal and oropharyngeal regions as regards both the uvula and lingual vallecula. The uvula and lingual vallecula were moved in the posterior direction.

\section{References}

1. Demetriades N, Chang DJ, Laskarides C, Papageorge M. Effects of mandibular retropositioning, with or without maxillary advancement, and oro-naso-pharyngeal airway and development of sleep related breathing disorders. J Oral Maxillofac Surg. 2010; 68: 2461-6.

2. Hwang $\mathrm{S}$, Chung $\mathrm{CJ}$, Choi YJ. Huh JK, Kim KH. Changes of hyoid, tongue and pharyngeal airway after mandibular setback surgery by intraoral vertical ramus osteotomy. Angle Orthod. 2010; 80: 302-7.

3. Aydemir H, Memikoðlu U, Karasu H. Pharyngeal airway space, hyoid bone position and head posture after orthognathic surgery in Class III patients. Angle Orthod. 2012; 82: 993-1000.

4. Becker OE, Avelar RL, Göelzer JG, Dolzan Ado N, Haas OL Jr, De Oliveira RB. Pharyngeal airway changes in Class III patients treated with double jaw orthognathic surgery-maxillary advancement and mandibular setback. Oral Maxillofac Surg. 2012; 70: 639-47.

5. Becker OE, Avelar RL, do N Dolzan A, Haas OL Jr, Scolari N, de Oliveira RB. Soft and hard tissue changes in skeletal Class III patients treated with double-jaw orthognathic surgery-maxillary advancement and mandibular setback. Int J Oral Maxillofac Surg. 2014; 43: 204-12.

6. Ronchi P, Cinquini V, Ambrosoli A, Caprioglio A. Maxillomandibular Advancement in obstructive sleep apnea syndrome patients: a restrospective study on the sagittal cephalometric variables. J Oral Maxillofac Res. 2013; 4: e5. eCollection 2013.

7. Hong JS, Park YH, Kim YJ, Hong SM, Oh KM. Three-dimensional changes in pharyngeal airway in skeletal Class III patients undergoing orthognathic surgery. Oral Maxillofac Surg. 2011; 69: 401-8.

8. Lee Y, Chun YS, Kang N, Kim M. Volumetric changes in the upper airway after bimaxillary surgery for skeletal class III malocclusions: a case series study using 3-dimensional cone-beam computed tomography. J Oral Maxillofac Surg. 2012; 70: 2867-75.

9. Yuan L, Shen G, Wu Y, Jiang L, Yang Z, Liu J, et al. Three-dimensional analysis of soft tissue changes in full-face view after surgical correction of skeletal Class III malocclusion. J Craniofac Surg. 2013; 24: 725-30. 
10. Kim MA, Kim BR, Choi JY, Youn JK, Kim YJ, Park YH. Threedimensional changes of the hyoid bone and airway volumes related to its relationship with horizontal anatomic planes after bimaxillary surgery in skeletal Class III patients. Angle Orthod. 2013; 83: 623-9.

11. Abdelrahman TE, Takahashi K, Tamura K, Nakao K, Hassanein KM, Alsuity A, et al. Impact of different surgery modalities to correct class III jaw deformities on the pharyngeal airway space. J Craniofac Surg. 2011; 22: 1598-601.

12. Hasebe D, Kobayashi T, Hasegawa M, Iwamoto T, Kato K, Izumi N, et al. Changes in oropharyngeal airway and respiratory function during sleep after orthognathic surgery in patients with mandibular prognathism. Int J Oral SurgMaxillofac. 2011; 40: 584-92.

13. Pereira-Filho VA, Castro-Silva LM, de Moraes M, Gabrielli MF, Campos $J A$, Juergens P. Cephalometric evaluation of pharyngeal airway space changes in Class III patients undergoing orthognathic surgery. J Oral Maxillofac Surg. 2011; 69: 409-15.

14. Oktay $\mathrm{H}$, Ulukaya E. Maxillary protraction appliance effect on the size of the upper airway passage. Angle Orthod. 2008; 78: 209-14.

15. Kitagawara K, Kobayashi T, Goto H, Yokobayashi T, Kitamura N, Saito $C$. Effects of mandibular setback surgery on oropharyngeal airway and arterial oxygen saturation. Int J Oral Maxillofac Surg. 2008; 37: 328-33.

16. Iwasaki T, Saitoh I, Takemoto Y, Inada E, Kakuno E, Kanomi R, et al. Tongue posture improvement and pharyngeal airway enlargement as secondary effects of rapid maxillary expansion: a cone-beam computed tomography study. Am J Orthod Dentofacial Orthop. 2013; 143: 235-45.

17. Muto T, Yamazaki A, Takeda S, Sato Y. Effect of bilateral sagittal split ramus osteotomy setback on the soft palate and pharyngeal airway space. Int J Oral Maxillofac Surg. 2008; 37: 419-23.

18. Shigeta Y, Ogawa T, Tomoko I, Clark GT, Enciso R. Soft palate length and upper airway relationship in OSA and non-OSA subjects. Tex Dent J. 2013; 130: 203-11.

19. Chen F, Terada K, Hanada K, Saito I. Predicting the pharyngeal airway space after mandibular setback surgery. J Oral Maxillofac Surg. 2005; 63: 1509-14.

20. Xiao $Y$, Chen X, Shi H, Yang $Y$, He L, Dong J, et al. Evaluation of airway obstruction at soft palate level in male patients with obstructive sleep apnea/hypopnea syndrome: dynamic 3-dimensional CT imaging of upper airway. J Huazhong Univ Sci Technolog Med Sci. 2011; 31: 413-8.

21. Bilston LE, Gandevia SC. Biomechanical properties of the human upper airway and their effect on its behavior during breathing and in obstructive sleep apnea. J Appl Physiol (1985). 2014; 116: 314-24.

22. Liukkonen M, Vähätalo K, Peltomäki T, Tiekso J, Happonen RP. Effect of mandibular setback surgery on the posterior airway size. Int J Adult Orthodon Orthognath Surg. 2002; 17: 41-6. 